\title{
QUALE PANORAMA SULLA LETTERATURA GERMANOFONA NEL 2017?
}

WHICH PANORAMA ON THE GERMANOPHONE LITERATURE IN 2017?

Héloïse Elisabeth Marie-Vincent Ghislaine Ducatteau

\section{Parole chiave:}

Letteratura, storia, canonicità, germanofonia
KeYwORDS:

Literature, history, canonicity, germanophony 
Meid, Volker (2017). Das Buch der Literatur. Deutsche Literatur vom frühen Mittelalter bis ins 21. Jahrhundert. Reclam.

La prima copertina potrebbe essere fuorviante perché appare solo il titolo - senza il sottotitolo che poi apparisce alla pagina 3. Strategia di marketing per colpire l'interesse di tutti gli amici della letteratura qualsiasi i generi o la lingua originale o semplice omissione? Suggerisce che il libro abbraccia la letteratura generale nel suo complesso, in altre parole, la letteratura mondiale o la letteratura globale per adottare il vocabolo più attuale. La quarta copertina chiarisce che si tratta di letteratura tedesca, questa volta troppo restrittivamente, poiché questa enciclopedia dà un posto significativo alla letteratura svizzera tedesca, austriaca e inserisce persino riferimenti alle arti francesi, spagnole, italiane, greche e russe. Il primo lembo va nella stessa direzione della quarta copertura. La prefazione oscilla tra denominazioni diverse per quanto riguarda l'oggetto del libro: letteratura tedesca, letteratura in lingua tedesca, letteratura dell'area linguistica tedesca, riflesso della tensione epistemologica e didattologica. Prova di questo modo il ritardo delle letterature tedescofone rispetto alle letterature francofone o anglofone che si sono affermate.

Il posto destinato alla letteratura germanofona fuori della Germania deluderà più di uno. Il principale autore austriaco, Stefan Zweig (da non confondere con Arnold Zweig, che è presente) è assente dall' intero compendio. Ciò è tanto più sorprendente in quanto un paragrafo è incentrato sul genere del racconto di cui è riuscito a diventarne il maestro, senza dimenticare le numerose pubblicazioni recenti a proposito di lui riunite sul sito dell'Associazione internazionale Stefan Zweig basata a Salisburgo: vengono scritte tanto da studiosi latinoamericani che anglosassoni rivelando l'interesse non esaurito per il «Mittler in der Weltliteratur » (Zohn, 1964, p. 19), chiamato anche il " lance[ur] de ponts » (Delatte, 2006, p. 7) o « Penseur de l'Europe» (Charton, 2012, p. 54). Gli scrittori canonici germanofoni svizzeri presenti in una storia della letteratura svizzera (Rusterloz y Solbach, 2007) sono lasciati fuori dal presente libro: Albert Bitzius, Felix Moeschlin, Meinrad Inglin...

La struttura del compendio è classica: cronologica. Dopo tre capitoli che imbracciano periodi soprasecolari, i sei capitoli seguenti corrispondono a un secolo in particolare, all'eccezione del settimo capitolo dedicato alla rivoluzione e alla restaurazione. Ognuno ha anche un colore che si trova sulla grondaia e permette di individuare senza nemmeno aprire il libro. La prima parte richiama l'attenzione sull'Alto Medioevo con un'attenzione ai rapporti tra orale e scritto, elemento chiave per familiarizzarsi con le glosse e la letteratura in alto sassone. Il secondo capitolo sul Pieno Medioevo spiega lo sviluppo dell'idea di autore, lo svolgimento dell'amore cortese. Il terzo sul Basso Medioevo annuncia il seguente dacché, accanto a considerazioni su novità generiche, il romanzo arturiano e la fiaba-novella, mostra come la scienza si contrappone alla 
religione. Il quarto sul Cinquecento mette in relazione la prima Bibbia tedescofona con le università, la letteratura neolatina e il medio del volantino che favorisce la diffusione delle idee e delle incisioni. Il quinto associa il barocco al manierismo fermandosi sul sonetto. Il sesto ci sensibilizza allla riforma teatrale, al rococò e alla robinsonata. Il settimo sottolinea l'importanza del conto, del calendario e dell'almanacco in questo periodo. L'ottavo accenna sul movimento «Giovane Germania » intorno a Heinrich Laube e Theodor Mundt. L'ultimo dedicato al Novecento è di gran lunga il più sostanzioso e il più pan-tedesco. È l'unico a dedicare interi sottocapitoli ad autori svizzeri, austriaci e cechi: Hugo von Hofmannstahl, Kafka per limitarsi ai più mediatizzati. E l'unico a trattare degli scrittori e delle scrittrici Aussiedler*innen (quei/quelle tedeschi/tedesche nati/nate al di fuori dei confini dell'attuale Germania, nei territori dell'Impero tedesco) tra i quali Christa Wolf è probabilmente una delle più didattizzate. Benché Screenshot di adattamenti teatrali e cinematografici appaiano nei capitoli precedenti con Nathan der Weise, Effi Briest, logicamente - se ci basiamo sull'accento della germanistica accordato alla Neue Deutsche Literaturwissenschaft, cioè alla letteratura dell'ultimo secolo - è l'ultimo capitolo che ne riunisce di più: Homo faber, Der Besuch, Jahrestage... Le arti visive statiche vi occupano ancora un posto significativo, che si tratti di poesia visiva, pittura e incisione.

Attardiamoci sulla struttura generale che si vuole particolarmente trasversale. Una delle peculiarità del compendio è la rinuncia alle note a piè di pagina. Invece, tutte le pagine all'interno dei capitoli contengono informazioni sui margini, meno faticoso per l'occhio: didascalie di immagini, biografie di sceneggiatori, definizioni concettuali, sinossi di libri, citazioni lunghe in inserti colorati. Se queste ultime sono in tedesco antico-alto tedesco o medio-alto tedesco, vengono presentate con la loro traduzione in tedesco moderno. Alla fine del libro, le indicazioni bibliografiche, una tabella di illustrazioni e testi, un indice di tutte le persone (sia autori, autrici, parenti di loro, artisti correlati ed artiste correlate) menzionate e un più sintetico dei paragrafi (che occupano tutti due pagine dentro i capitoli) facilitano la consultazione.

Il prontuario è innovativo in termini di scelta degli autori e dei movimenti scelti? Fermiamoci sul canone letterario ritenuto già prima di aprire il libro. Non sorprende che l'autore evidenziato dalla prima copertina, attraverso una fotografia della sua casa di Weimar e una parte del suo ritratto realizzato da Joseph Karl Stieler, sia Goethe. Questa prima copertina presenta anche due elementi meno emblematici delle lettere germaniche: un pezzo del manoscritto di Sarah Kirsch e uno del manoscritto di Alram von Gresten. Si può definire questo testo come enciclopedia? I testi selezionati in antico tedesco sono ben all'interno del canone letterario stabilito dalla stessa casa editrice: l'Abrogans, Notker il tedesco, traduttore di Boezio, Otfrid di Weißenburg per riprendere i più emblematici. Meid dedica un sottocapitolo alla prima poetessa 
tedesca assente dall'antologia bilingue di Stephan Müller (2007), sottolineando così una riabilitazione della produzione artistica femminile. Diversi/diverse poeti/poetesse presenti in una delle antologie poetiche più famose (Echtermeyer, 2010) sono assenti dal Volker Meid: Heinrich von Veldecke, Heinrich von Morungen, Neidhart von Reuental... Tuttavia, i più famosi sono presenti nei tre libri citati: Hartmann von Aue, Walther von der Vogelweide... È un peccato che Christine Benedikte Naubert, l'autrice dei primi romanzi storici in tedesco, non sia menzionata. Lo stesso vale per Gertrud Kolmar, morta ad Auschwitz, che era presente nell'opera di Eschenmeyer ma era assente nell'opera di Meid.

In conclusione, questa sintesi della storia letteraria di lingua tedesca è adatta sia ai non specialisti e non specialiste sia agli intenditori e alle intenditrici fuori della sfera accademica per scoprire non solamente autori o libri poco conosciuti e autrici poco conosciute ma anche le condizioni che hanno permesso la loro emergenza, cioè sia istituzioni, sia movimenti di idee. Un codice QR che fornirebbe l'accesso a documentazione aggiuntiva sarebbe stato il benvenuto. Garantirebbe l'accesso a video e articoli di qualità per chi progetta pubblicazioni ulteriori.

\section{REFERENCIAS BIBLIOGRÁFICAS}

Charton, Arianne (2012) Stefan Zweig ou « l'esprit de l'Europe ». Villa Europa, 3, Universitätsverlag Saarbrücken, 47-54.

Delatte, Anne-Élise (2006) Übersetzer der Geschichte, Übersetzungsgeschichten: drei biographische Schriften Stefan Zweigs übertragen von Alzir Hella (Fouché, Marie Antoinette, Maria Stuart), Unis Nantes/Düsseldorf.

Echtermeyer, Ernst Theodor (2010). Deutsche Gedichte. Elisabeth K. Paefgen y Peter Geist (Eds.), Cornelsen.

(2007). Althochdeutsche Literatur. Eine kommentierte Anthologie. Zweisprachig. trad. y ed. por Stephan Müller, Reclam.

Rusterholz, Peter y Solbach, Andreas (2007). Schweizer Literaturgeschichte. Metzler.

Zohn, Harry (1964) Wiener Juden in der deutschen Literatur. Olamenu. 